\title{
TWO-COLOR OBSERVATIONS OF RR LYRAE STARS IN M14
}

\author{
AMELIA WEHLAU and NICHOLAS POTTS \\ University of Western Ontario, London, Canada
}

\begin{abstract}
Two-color photographic photometry has been carried out on RR Lyrae variables in M14. The position of these variables in the color-magnitude diagram is shown, and it is noted that there is a lack of horizontal branch stars to the red of the variable star gap. Color-amplitude, periodamplitude and color-period relations for the RR Lyrae variables measured are shown.
\end{abstract}

M14 (NGC 6402, $\alpha=17^{\mathrm{h}} \cdot 35^{\mathrm{m}} .0, \delta=-3^{\circ} 15^{\prime}, l^{\mathrm{II}}=21^{\circ}, b^{\mathrm{II}}=+14^{\circ}$ ) is a cluster of intermediate metal content, placed in class IV by Morgan (1959). It has a good number of variable stars, with 5 Population II Cepheids and more than 70 RR Lyrae variables, with periods determined for about 45 of the latter type (Sawyer Hogg and Wehlau, 1966, 1968). However it is not an easy cluster to study because it has a distance modulus of more than 15 magnitudes and in addition appears to have more than a magnitude absorption in the blue.

A series of 42 plates of this cluster was taken with the 48 -in. telescope of the University of Western Ontario from 1970 to 1972 and, in addition, 11 plates taken by Dr S. Demers in 1967 with the Naval Observatory 61-in. at Flagstaff, and 23 plates taken in 1952 by Dr H. Arp with the 60 and 100-in. telescopes at Mt. Wilson, were measured. Altogether the plate material consists of 33 blue and 43 visual plates of the cluster.

Two separate photoelectric sequences obtained by Dr Demers and Dr R. Racine were combined and used as one sequence with limiting magnitudes of 18.8 in $B$ and 17.8 in $V$ There appears to be no systematic differences between the two sequences.

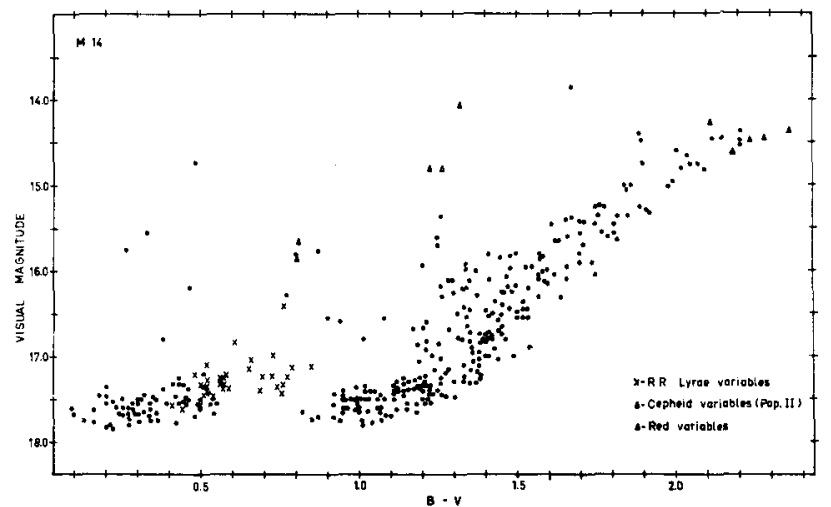

Fig. 1. Color-magnitude diagram for M14 showing the position of the variable stars. The nonvariable stars represent about four-tenths of the cluster area. All of the known Population II Cepheids and red variables are shown and about one-half of the known RR Lyrae variables. No stars below the limiting magnitudes of the sequence are shown. 


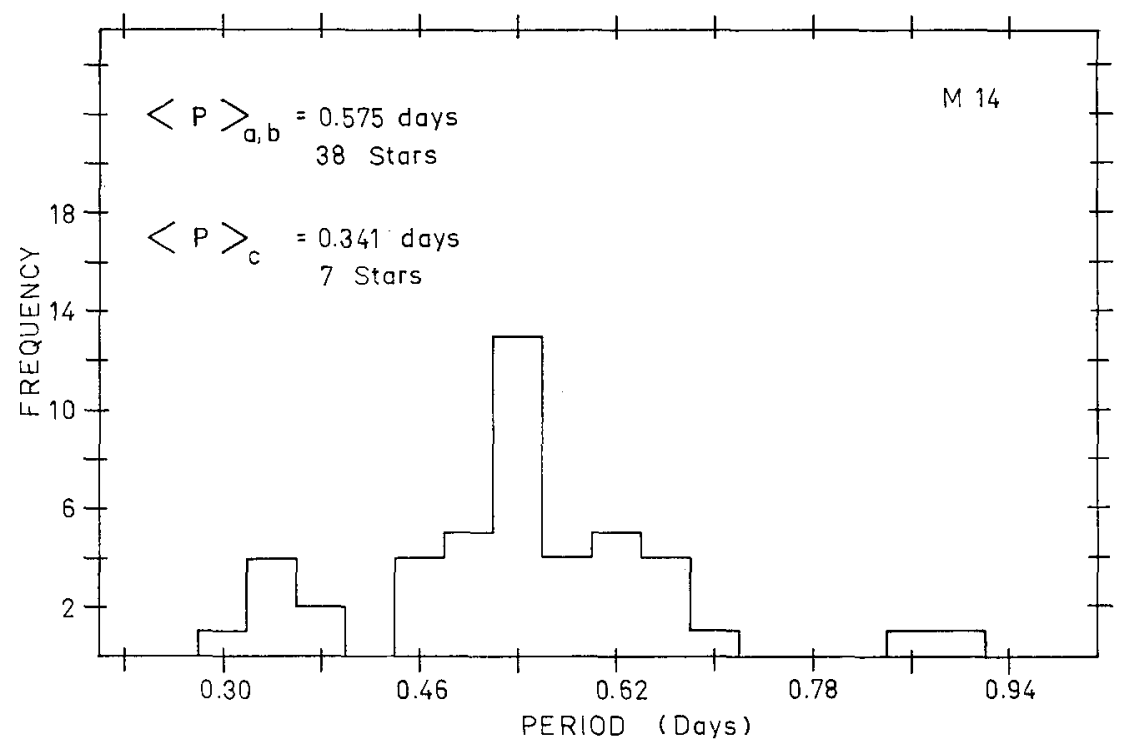

Fig. 2. Frequency of period histogram for RR Lyrae variables with known periods in M14.

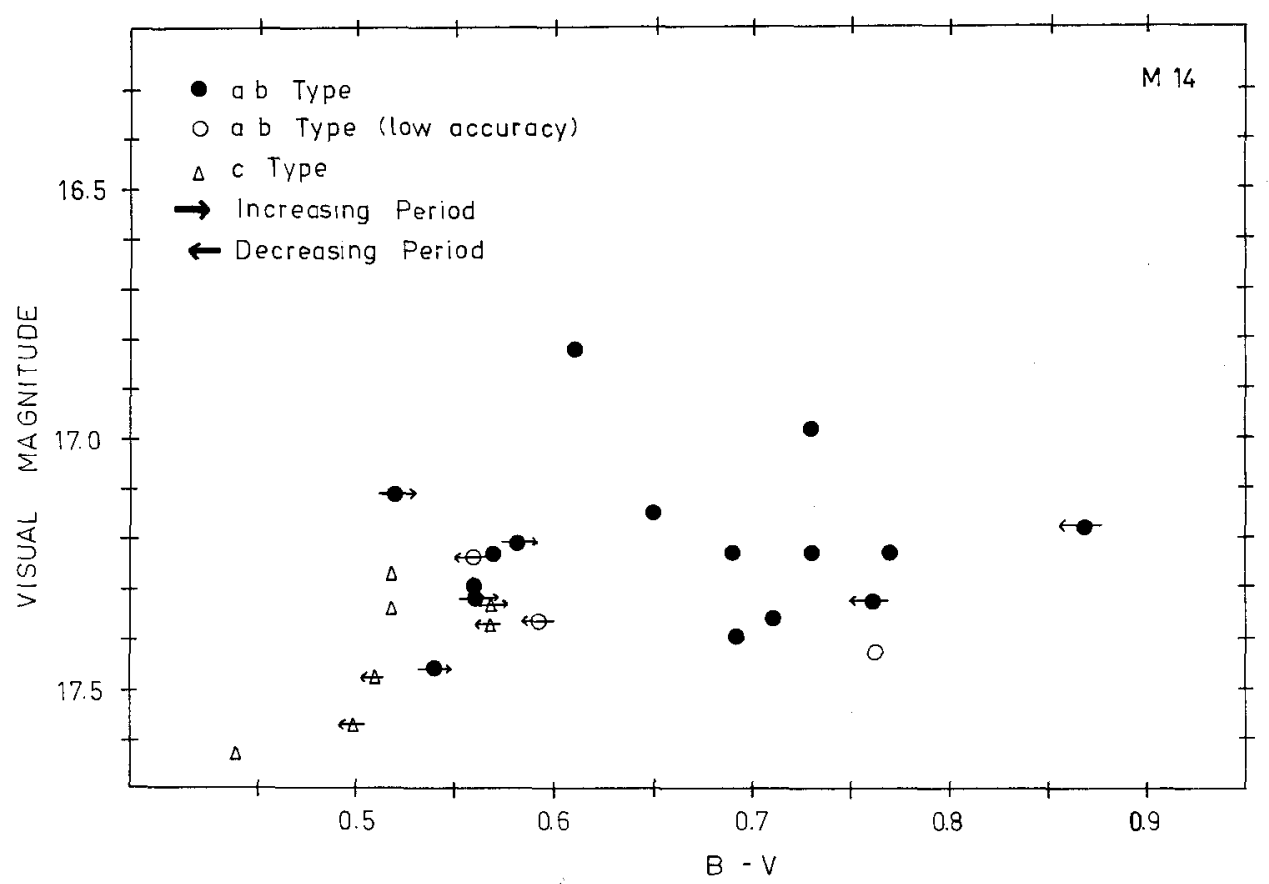

Fig. 3. The variable star region of the horizontal branch showing relation between color and period change. 


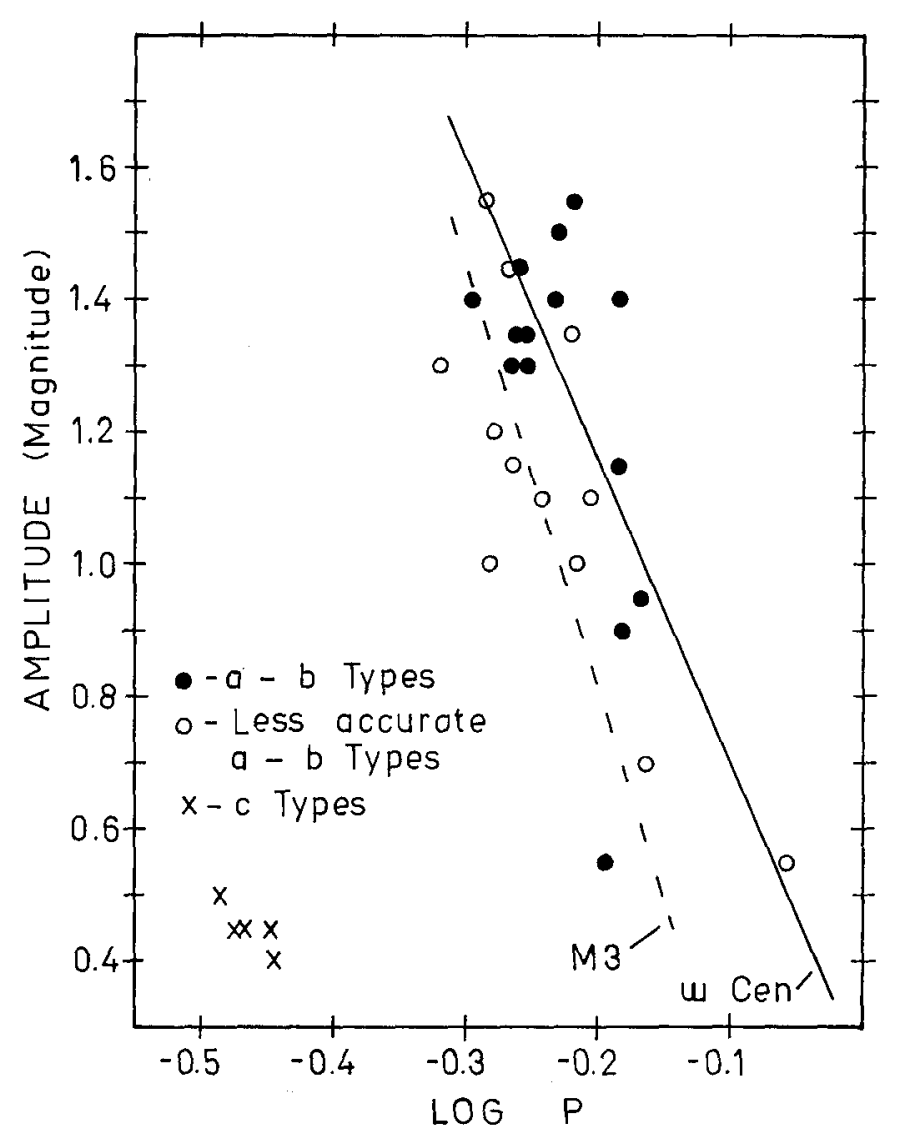

Fig. 4. Period-amplitude relation for RR Lyrae variables in M14. The positions of mean lines for variables in $\omega$ Cen and M3 are also shown.

Light curves were constructed in both colors for $25 a b$-type stars. These were transformed into intensity curves and integrated in order to obtain mean colors. Periods are known for $7 c$-type variables but we were unable to get satisfactory visual light curves for any of these. Being fainter visually than the $a b$-types they fall very close to the plate limit on the visual plates and in many cases there appeared to have been a period change between the sets of observations. However we did have enough observations in both colors to obtain somewhat less accurate values of $B-V$ for them as well as other $a b$-type variables.

Figure 1 shows a color-magnitude diagram for M14 recently determined by Christine Smith (1973), with the RR Lyrae variables included in the diagram. The nonvariable stars represent somewhat less than half the globular cluster. Stars not known to be variable lying in the $a b$-type portion of the variable star gap were investigated and in almost all cases were found to be either a blend of more than one image or to be varying. In this way 11 new variables have been discovered. Such stars in the 
c-type region have yet to be investigated. Because of their lower amplitudes their variability is more difficult to determine.

Because of this uncertainty as to which stars are variable the blue edge of the variable star gap is not accurately determined. There is an obvious lack of horizontal branch stars to the red of the gap. On the basis of metallicity and Oosterhoff group, such a lack would not have been expected.

A good value for the reddening was not available for this cluster, so we decided to use the method developed by Sturch $(1966,1967)$, which uses the colors of $a b$-type RR Lyrae stars during minimum. This method requires a knowledge of the ultraviolet excess which is unknown for this cluster. However there appears to be a relationship between the frequency distribution of RR Lyrae periods in a cluster and the ultraviolet excess. The frequency distribution for M14 is that of an Oosterhoff group I cluster and is shown in Figure 2. On the basis of this distribution a value of $\delta$ of $0.08 \mathrm{mag}$. was used to obtain a reddening of $0.35 \mathrm{mag}$. This is considerably lower than values obtained by others using the integrated color of the cluster.

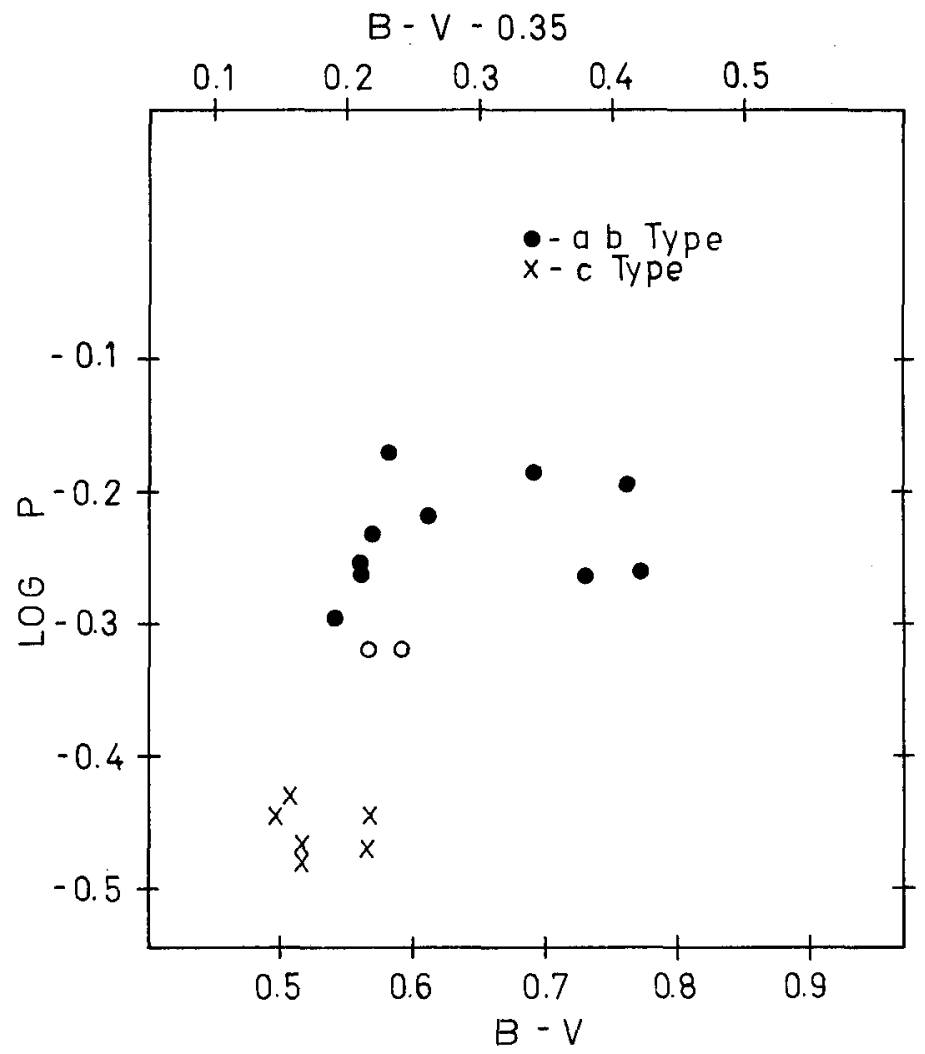

Fig. 5. Period-color relation for RR Lyrae variables with the most accurate colors in M14. The open circles are less accurate colors for variables 14 and 33 which have periods near 0.47 , the transition period for this cluster. 
Figure 3 shows the color-magnitude diagram for the RR Lyrae stars alone. The arrows show the sense of period change when such a change is observed. The observed changes appear to be sudden and random and not easily attributed to evolutionary changes.

A plot of period versus amplitude is shown in Figure 4. Mean lines for the variables in $\omega$ Cen (Dickens and Saunders, 1965) and M3 (Roberts and Sandage, 1955) are shown as well.

Figures 5 and 6 show the relationship between color and period and color and amplitude respectively. Only those $a b$-type variables which fall in completely uncrowded areas are plotted since colors obtained for the other variables appear to be reddened through proximity to red stars in the cluster.

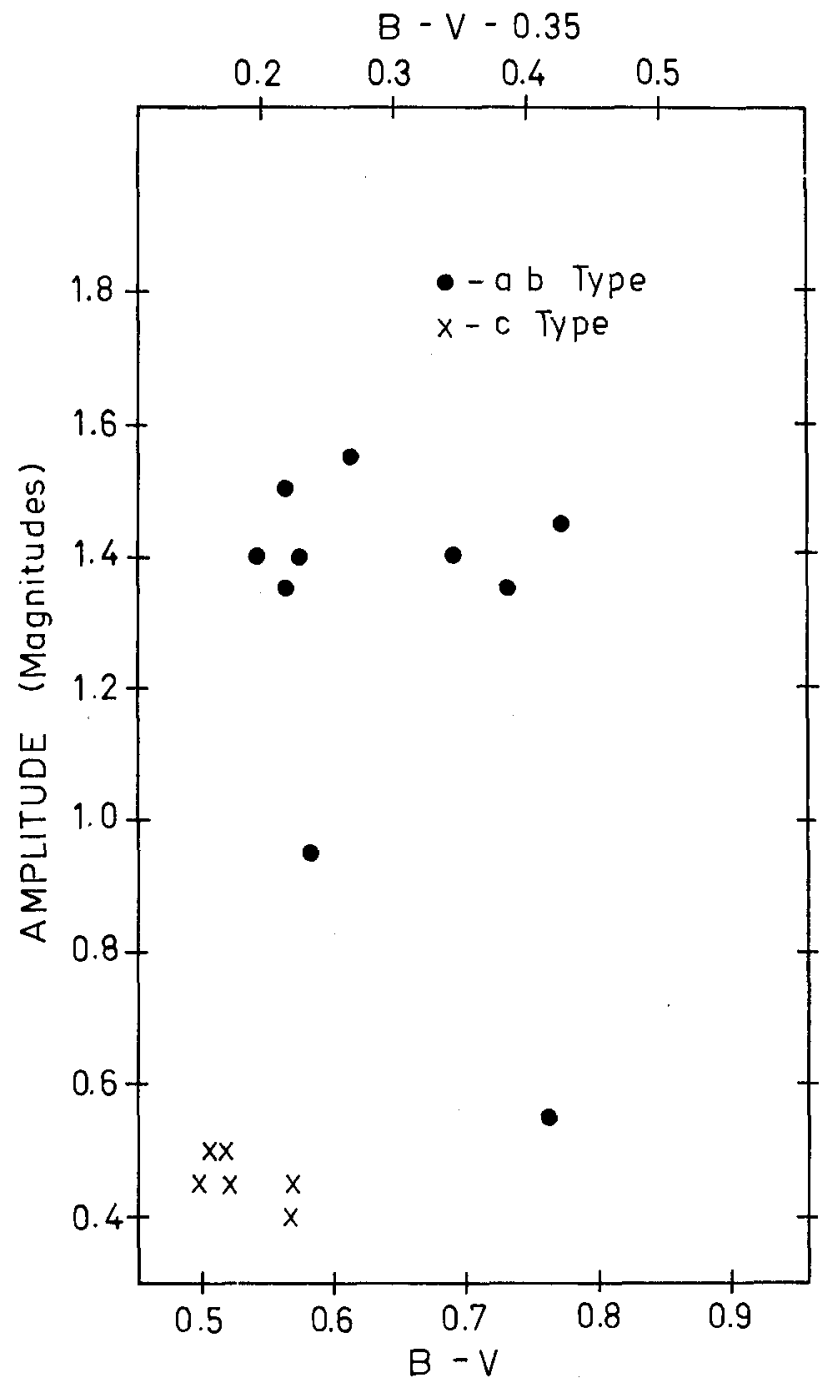

Fig. 6. Color-amplitude relation for RR Lyrae variables with the most accurate colors in M14. 


\section{References}

Dickens, R. J. and Saunders, J.: 1965, Roy. Obs. Bull. No. 101.

Morgan, W. W.: 1959, Astron. J. 64, 432.

Roberts, M. and Sandage, A.: 1955, Astron. J. 60, 185.

Sawyer Hogg, H. and Wehlau, A.: 1966, Publ. David Dunlap Obs. 2, No. 17.

Sawyer Hogg, H. and Wehlau, A.: 1968, Publ. David Dunlap Obs. 2, No. 19.

Smith, C.: 1973, unpublished M.Sc. thesis, University of Western Ontario.

Sturch, C.: 1966, Astrophys. J. 143, 774.

Sturch, C.: 1967, Astrophys. J. 148, 477.

\section{DISCUSSION}

Cox: What fraction of the stars in the variable gap do you feel are non-variable?

Wehlau: The fraction where the $a b$-types are found is probably very low as the stars that were thought to be non-variable have been looked at and so far all have turned out to be new variables or blended with another star image. There are more such stars found in that part of the gap where the $c$-type variables appear. However they may also turn out to be variable as it is much harder to recognize the variability of these fainter stars with smaller amplitude of variation. We plan to make a greater effort to check the constancy of these stars.

Schwarzschild: (To Mrs Hogg and Mrs Wehlau). Do you have any comment concerning the earlier history of the search for variables in this cluster?

Hogg: I discovered 72 variables in this cluster from plates at the Dominion Astrophysical Observatory in the 1930's. Then in recent years Dr Amelia Wehlau has been collaborating with me on the the determination of periods. We have already published forty and have some others determined, but not published. Dr Wehlau discovered four more variables and one nova. 